\title{
Wenn die Kamera dunkel bleibt - Psychodramatische Überlegungen zur Rolle digitaler Medien beim Lehren und Lernen.
}

\author{
Karsten Krauskopf
}

Angenommen: 6. Mai 2021 / Online publiziert: 18. Juni 2021

(C) Der/die Autor(en) 2022

Zusammenfassung Dieser Beitrag der Zeitschrift für Psychodrama und Soziometrie (ZPS) entwickelt aus psychodramatischer Perspektive die These, digitale Medien nicht nur als Lernwerkzeuge auf einer kognitiven, sozialen und handlungsnahen Ebene zu verstehen, sondern auch ihren Einfluss auf Begrenzung und Ermöglichung soziometrischer Wahlen in Lehr-Lern-Settings zu betrachten. Ausgehend von einem Einblick in aktuelle Konzepte der pädagogisch-psychologischen Forschung wird das Konzept des Aufforderungscharakters - Affordanzen - digitaler Medien eingeführt. Anhand einer Vignette aus dem hochschuldidaktischen Kontext werden mögliche „Verführungen“ durch Medien im Hinblick auf soziometrisches Handeln rekonstruiert und vor dem Hintergrund von Rollenerwartungen und Morenos Konzept der Begegnung diskutiert.

Schlüsselwörter Psychodrama · Unterrichtsforschung · Digitales Lernen · Corona $\cdot$ Hochschuldidaktik

Karsten Krauskopf ( $\square)$

c/o Fachhochschule Potsdam, Kiepenheuerallee 5, 14469 Potsdam, Deutschland

E-Mail: karsten.krauskopf@fh-potsdam.de 


\title{
When the screen stays dark: psychodramatic thoughts on the impact of digital media on learning processes
}

\begin{abstract}
This article in the German Journal Zeitschrift für Psychodrama und Soziometrie (ZPS) develops the argument that-from a psychodramatic perspective- that digital technologies cannot only be considered cognitive or social tools, which might augment teaching and learning processes. In addition, we should consider the impact of their affordances on our sociometric actions. Using a vignette from higher education the authors reflects on possible "temptations" afforded by the digital tools used. In conclusion, these considerations are discussed on the basis of role expectations and Moreno's concept of encounter.
\end{abstract}

Keywords Psychodrama · Digital teaching · Digital learning · Corona · Higher education

\section{Einleitung}

$\mathrm{Zu}$ Beginn einige Worte zu dem was in der Regel aktuell in der pädagogisch-psychologischen Forschung unter Unterricht verstanden wird. Verbreitet ist folgendes Verständnis: Unterricht wird als kompetenzorientiert begriffen, d.h. SchülerInnen soll es möglich sein, Kompetenzen in den Fächern und zusätzlich überfachliche Kompetenzen zu erwerben (Klieme und Rakoczy 2008). Das was als Unterricht geschaffen wird (oder entsteht) wird nach Helmke (2009) als ein Angebots-NutzungsModell begriffen, in dem professionell kompetente Lehrkräfte (vgl. das Modell nach Baumert und Kunter 2013) ein Angebot gestalten, das von den heterogenen Lernenden (im Hinblick auf Vorwissen, Motivation, Lernstrategien, etc.) mehr oder weniger effektiv genutzt werden kann. Unterricht kann im Hinblick auf seine Qualität beurteilt werden. Aktuell relevante und durch empirische Forschung begründete Kriterien guten Unterrichts umfassen (1) gute Klassenführung, die durch Störungsprävention ausreichend Zeit für die Beschäftigung mit Lerninhalten ermöglicht, (2) ein unterstützendes und sozial orientiertes Klassenklima und (3) kognitiv aktivierende Aufgabe, die eine tiefere Verarbeitung des Stoffes anregen und nicht allein auf Faktenwissen ausgerichtet sind (Mayer, 2004, nach Klieme und Rakoczy 2008). Werden nun digitale Medien integriert, so werden diese allgemein dahingehend bedacht und geprüft, inwiefern sie, bzw. einzelne ihrer Eigenschaften, für diese Dimensionen guten Unterrichts zu- oder abträglich sind.

\subsection{Der Aufforderungscharakter digitaler Medien - Affordanzen}

Insgesamt ist zu sagen, dass Überblicksarbeiten zu im Durchschnitt positiven Effekten bezüglich des Einsatzes digitaler Medien kommen, wobei diese seit den 60ern mit der technologischen Entwicklung im Trend positiver ausfallen. Gleichzeitig streuen Effekte je nach Kontext breit (z.B. Kulik 2003). Es gibt jedoch eine zentrale Kontroverse in der Forschung zum digital gestützten Lernen (wobei es viele angrenzende Begriffe gibt, wie computervermittelt, computergestützt, medienbasiert, 
etc. auf deren nuancierte Unterschiede hier nicht eingegangen werden kann). Sie dreht sich um die Frage, ob digitale Medien in einem Lehr-Lern-Setting lediglich die ohnehin stattfindenden Prozesse mehr oder weniger unterstützen oder ob die Eigenschaften digitaler Medien, z. B. neuartige Visualisierungen, nicht doch genuin etwas Eigenes zum Lernprozess beitragen. In der sogenannten Clark-Kosma-Debatte vertrat Richard Clark (1983) die Position Medien seien - wie der Name sagt etwas, das Inhalte bereitstelle, vermittle und legte den Schwerpunkt darauf, dass Inhalte und Instruktionsmethoden einen größeren Einfluss auf das Lernen (den Lernerfolg) hätten. Dagegen fokussierte Robert Kozma (1994) auf den Umstand, dass manche Medien durch die ihnen eigenen Möglichkeiten ganz spezifische Aufgaben ermöglichten - und damit andersartige Auseinandersetzung mit Inhalten. Betrachtet man die Entwicklungen seither, insbesondere soziale Medien, fällt auf, dass diese digitalen Werkzeuge immer weniger eindeutigen Charakter haben. D.h. ein computerbasiertes Programm zum Vokabeln lernen charakterisiert heute weniger das, was wir unter einem digitalen (Lern-)Werkzeug verstehen. Diese werden heute vielmehr durch die Frage nach Algorithmen bestimmt, die Tweets oder Facebook-Posts ein- und ausblenden. Im Folgenden möchte ich daher die Position vertreten, dass es von besonderem Interesse ist, spezifische Eigenschaften eines Mediums im Sinne Kozmas (oder einer Gruppe diesbezüglich ähnlicher Medien) in den Vordergrund zu stellen und sich mit Fragen zu bezüglich des Potentials dieser Eigenschaften zur Umsetzung didaktischer Prinzipien beschäftigen.

Dazu erscheint es hilfreich, den Begriff der Affordanz einzuführen (engl. Affordances), der im Kern den Gedanken beschreibt, dass Gegenstände zu bestimmten Handlungen auffordern. Während dies ursprünglich im Zusammenhang mit der konkreten Welt verstanden wurde - ein Stuhl fordert zum Sitzen auf (Gibson 1982, S. 138) - hat sich dieser Begriff zu einem wichtigen Denkmodell zum Verstehen des Lehrens und Lernens mit zeitgenössischen digitalen Medien entwickelt (Angeli und Valanides 2018; Krauskopf et al. 2012; Valanides 2018). Grundlegend ist die Idee, dass digitale Technologien als Werkzeuge verstanden werden können, welche einige Aneignungsprozesse in Lehr-Lern-Settings fördern und andere behindern. D.h. sie sind ein bedeutsames etwas, das zwischen den Lernenden, Lehrenden und der ,Welt“ existiert. Bedeutsam sind sie dahingehend, dass sie nicht lediglich ihren NutzerInnen dienen, d. h. deren Verständnis und Willen in die Welt tragen, bzw. dies den anderen Personen vermitteln helfen. Sondern sie bringen gewissermaßen ihr eigenes (Un-)Vermögen ein. Besonders griffig lässt sich dies an den Arbeiten von Suthers und Hundthausen (z. B. Suthers 2006) erläutern: Ein Tabellenformat (Tabellenkalkulationsprogramm) ermöglicht es, Dinge in Zeilen und Spalten zueinander in Beziehung zu setzen. Es verführt aber auch dazu, dies zu tun. Und noch mehr; sie verführt dazu, es vollständig zu tun, d.h. es kann sein, dass man als NutzerIn davorsitzt und sich bemüht, eigentlich sinnlose Zellen zu füllen, weil sie eben da und noch leer sind.

\subsection{Mentale Modelle digitaler Medien}

Dieses Phänomen wird besonders komplex, wenn zum einen die geplanten Möglichkeiten über klare Funktionen wie eine „,Tabellenkalkulation“ hinausgehen. Insbeson- 
dere Soziale Medien umfassen Einheiten, deren Grenzen fluide sind und durch die sogenannte „Appification“ zudem erweiterbar und individuell anpassbar sind. Was also ist z. B. die Funktion von Facebook? Mit Menschen in Kontakt bleiben? Neue Menschen kennenlernen? Sich politisch informieren? Politisch zu partizipieren? Die schönsten Momente aus dem eigenen Leben mit „der Welt“ teilen? Narzisstische Gratifikation erhalten? ... Sicher auch all das. Wir können also einerseits davon ausgehen, dass die Funktionalitäten digitaler Technologien spezifisches Denken, Fühlen und soziales Miteinander erzeugen - weil es ohne sie eben anders ist - und gleichzeitig wissen wir nicht wirklich, was da erzeugt wird und wozu wir aufgefordert oder gar verleitet werden. Begründen lässt sich dies nicht nur darin, dass es so vieles gibt, sondern dass es sich um emergente Phänomene handelt, die zusätzlich fluide sind. Ein simples Beispiel erscheint mir, wie im Verlauf der Corona-Pandemiemaßnahmen sich durch die steigende die Nutzung unterschiedlicher Videokonferenz-Tools diese sich plötzlich zu verändern begannen. Wo man vorher nur wenige Personen auf einmal sehen konnte, vermehrten sich plötzlich die „Kacheln“. Und gleichzeitig blieben sie unterschiedlich; wo das eine die spontane Bildung von „Kleingruppen“ unterstützt, bietet das andere dauerhaft parallele „Kanäle“ für die Kleingruppen. Dass diese fehlende lehr- und lernbezogene Eindeutigkeit die Nutzung in formalen Settings (Schule und Hochschule vor allem) insbesondere herausfordert, wird dabei schon länger diskutiert (z. B. Mishra und Koehler 2006).

Vor diesem Hintergrund bekommen aus pädagogischer Sicht Fragen nach LehrLernzielen und aus psychologischer Sicht das individuelle Verständnis des jeweiligen digitalen Werkzeugs Gewicht. Es kann also eine gegenseitige Beeinflussung angenommen werden von dem was ein digitales Medium theoretisch alles ermöglicht bzw. verhindert und dem welche Lernprozesse in dem jeweiligen Setting begünstigt werden sollen. Hierfür bietet sich das kognitionspsychologische Konzept des mentalen Modells an (Angeli und Valanides 2018; Krauskopf et al., 2012, 2015, 2020; vgl. auch Johnson-Laird 1983). Mentale Modelle werden als analoge Wissensrepräsentationen verstanden, in denen nicht nur einzelne (Wissens-)Elemente, sondern vor allem auch deren funktionale Beziehungen zueinander abgebildet sind. Ein Beispiel aus der lernpsychologischen Forschung ist hier das Verständnis des Herz-KreislaufSystem von SchülerInnen, das diese aus Lehrbuchtexten generieren und das durch deren Zeichnungen erfasst wird (Chi 2000). Anders als bei dem oben angeführten Beispiel der Tabelle, die durch ihre Kerneigenschaften alleine dazu verleitet, jede Zelle für sinnvoll ausfüllbar zu halten, ist es also bei komplexen digitalen Medien zusätzlich so, dass es davon abhängt, welches mentale Modell jeweils NutzerInnen davon konstruiert haben. Die mentalen Modelle braucht es, um zu verstehen, welchen Aufforderungscharakter das Medium für den einzelnen entfaltet. Greifen wir zurück auf die Idee des Angebots-Nutzungsmodells nach Helmke, so muss man davon ausgehen, dass die mentalen Modelle sowohl der Lehrenden als auch der Lernenden bezüglich der verwendeten digitalen Medien einen Einfluss darauf haben, was im „Unterricht“ letztlich geschieht. Da die Lehrenden jedoch in der Regel als spezifisch für das Lehren gebildete Fachkräfte verstanden werden, stehen insbesondere deren mentale Modelle, bzw. Kompetenzen im Umgang mit digitalen Medien im Vordergrund von Forschungsbemühungen. 


\section{Die Rolle der Lehrenden}

Zumeist wird Lehrenden eine ExpertInnen-Rolle zugeschrieben und Überlegungen zu den notwendigen medienbezogenen Kompetenzen von Lehrkräften werden angestellt. Hier lassen sich drei Hauptaspekte ausmachen (vgl. Blömeke 2000):

- Technische Kompetenzen und die eigene Erfahrung im Umgang mit digitalen Medien

- Mediendidaktische Kompetenzen für den Einsatz digitaler Medien zur Vermittlung von fachbezogenen Inhalten

- Medienerzieherische Kompetenzen, die sich auf die Thematisierung digitaler Medien als Unterrichtsgegenstand beziehen und die Vermittlung ethischer Normen und Werte im Umgang mit Medien einschließen.

Verstehen wir Rolle hier im Sinne Morenos als durch private und kollektive Seiten bestimmt (Moreno 1961, S. 520), so wird besonders deutlich, dass auch die mentalen Modelle der Lehrenden aus Erfahrungen als private Medien-NutzerInnen mit denen aus professioneller Nutzung zunächst nebeneinander stehen. So zeigt beispielsweise die Befragung von Lehramtsstudierenden, dass bei möglicher Mehrfachnennung über $40 \%$ YouTube als Unterhaltungsmedium verstehen und über $30 \%$ es als Informationsquelle sehen (Krauskopf et al. 2012). Fragt man weiter, werden noch andere Aspekte beschrieben, z. B. die Möglichkeiten der aktiven Nutzung durch Lernende. Dieser Ausschnitt soll illustrieren, dass wir uns vorstellen können, wie hier Erfahrungen auf YouTube als Nach-Entspannung-Suchende im Kontrast stehen mit solche als den-Satz-des-Pythagoras-verstehen-Wollende.

Einen Ausweg, der die Notwendigkeit anerkennt, dass es sowohl für Lehrende als auch Lernenden gilt, unterschiedliche Anforderungen und Erfahrungen zu integrieren scheint mir das verbreitete Narrativ des Wandels von der Lehrkraft zum Lern-Coach zu sein. Dies impliziert nicht unbedeutende Rollenanforderungen im Zusammenhang mit Lehr-Lern-Settings. Nicht unbedeutend, weil sie mit etablierten Anforderungen an Lehrkräfte nicht korrespondiert. Personen, die ein Fach- und Pädagogikstudium mit einem Selbstverständnis als UnterrichtsexpertInnen verlassen haben sollen nun digital unterstützen, begleiten, erweitern (augmentieren). An dieser Stelle möchte ich darauf fokussieren, dass hier die Zunahme von Komplexität der digitalen Medien selbst mit der Komplexität von Anforderungen an die Personen einhergeht, die diese Medien einhegen sollen.

Stellen wir uns also eine Lehrkraft vor, die entscheiden soll, ob sie eine Chatgruppe mit ihren SchülerInnen auf ihrem Smartphone einrichtet. Die Frage des Datenschutzes ist nur eine und lösbar. Was bedeutet dieser pausenlos potenziell vorhandene Kontakt aber innerlich für die Beteiligten? Will ich meinE LehrerIn „,in meinem Telefon“ bei mir haben? Will ich meine SchülerInnen immer „bei mir tragen"? Welche persönlichen Erwartungen sind daran geknüpft und welche kollektiven? Wenn es die KollegInnen auch tun, gehört es dann zum „Lern-Coach“-Dasein. Und nicht zuletzt hat die Corona-Pandemie ein Brennglas auf die Problematik der Ungleichheit gehalten. Kann ich als lehrende Person etwas etablieren, zu dem nicht alle einen Zugang haben? Soziometrisch gedacht lässt sich sagen: Das ist bereits der Alltag. Neben Ergebnissen die zeigen, dass SchülerInnen mit diagnostizierten 
Förderbedarfen soziometrisch häufiger isoliert sind (s. Krauskopf und Teke 2018), müssen wir uns eingestehen - so meine These, dass die Überlegungen zur Chatgruppe nur aufdecken, was im Klassenzimmer immer wieder ignoriert werden kann, nämlich dass einige (immer wieder) abgewählt werden. Und das heißt nicht, dass böse Lehrkräfte arme SchülerInnen sozial ausschließen. Nein. Plötzlich sehen hier alle, wer in der Chatgruppe nicht dabei sein kann, sich nicht beteiligt oder Schwarz auf Weiß abgewählt wird.

In dieser Form bezieht sich die Sorge, einzelne (oder Gruppen) systematisch an- und abzuwählen vor allem auf den schulischen Kontext. Wie aber sieht es in der Hochschule aus? Zu dieser Frage ließen sich Bücher schreiben. Da Artikel 5 Absatz 3 des Grundgesetzes die Freiheit von Forschung und Lehre garantiert, ist es leichter, sich an Hochschulen einerseits klar auf die Rolle als VertreterIn des eigenen Fachs und der eigenen Forschung zurückzuziehen. Und es ist tatsächlich eine wichtige Rollenerwartung, nicht nur als Lehrperson aufzutauchen und fachliche Inhalte für Studierende aufzuschließen, sondern auch ein Setting herzustellen, in dem Studierende in das Fach hineinsozialisiert werden (können). Andererseits werden Studierende, im Vergleich zu SchülerInnen, als Personen verstanden, die bereits eine Wahl getroffen haben. Sie werden als „freiwillig“ beteiligt gedacht; wenn schon nicht in der jeweiligen Veranstaltung doch wenigsten im jeweiligen Studiengang.

\section{Eine Corona-Vignette: Psychodramaseminar im Online-Format}

Psychodrama an einer Hochschule zu lehren heißt also, als PsychodramatikerIn soziometrisch und psychodramatisch zu handeln und somit als PraktikerIn aufzutauchen. Es heißt aber auch als (Hochschul-)DidaktikerIn zu handeln und Formen zu finden, in denen gelernt werden kann. Learning by doing - aber wie? Darüber zu berichten, wie man Psychodrama lehrt, wäre an dieser Stelle wohl weniger spannend, wenn durch die Corona-Pandemie nicht eine Situation entstanden wäre, die einen rein virtuellen Kontakt zu den Studierenden geschaffen hätte. Dies wiederum bietet einen Anlass über die oben beschriebenen Fragen des Aufforderungscharakters digitaler Technologien nachzudenken und zu skizzieren, wie dies Rollenanforderungen an die dozierende Person unter das Vergrößerungsglas rücken kann. Dazu folgende Überblicks-Vignette.

\subsection{Die Rahmenbedingungen}

Die kurzfristig beschlossenen Maßnahmen seitens der Hochschule lassen es nicht zu, das Seminar wie geplant als Block im Präsenzformat durchzuführen. Ich muss digital improvisieren. Was mir nach Lektüre der Arbeiten von Jakob Gershoni (z. B. 2016) wichtig geworden und geblieben ist, ist die Erwärmung anhand aufeinander aufbauende Aktionssoziometrie. In der Arbeit mit pädagogischen Fachkräften verwende ich dabei ein jeweils passendes Kompetenzmodell, das die Kriterien für die selbst- und fremdeingeschätzten Stärken darstellt (Krauskopf 2019). Zwei digitale Medien stehen im Zentrum der nun digitalen Version. Ein Videokonferenztool, das einen Hauptraum hat, in dem Präsentationen oder alle Teilnehmenden in kleinen 
Kacheln sichtbar sind, die Möglichkeit existiert, spontan Teilgruppen in eigene virtuelle Räume zuzuordnen, und einer Chat-Funktion, in der man für alle sichtbare und individuelle, ,private“ Nachrichten schreiben kann. Zusätzlich verwende ich eine Präsentations-Software, die auch die als Server-Version die Möglichkeit zur synchronen Online-Kollaboration beinhaltet. Zum einen für meine Erläuterungen, zum anderen die Server-Version dieses Programms als eine Art digitale Tischbühne. Aus der Teilnehmerperspektive hatte ich die Möglichkeit erlebt, dass sich die Gruppe über das Videotool austauscht, während alle gleichzeitig über den Browser auf eine Online-Präsentation zugreifen, die dann alle gleichzeitig bearbeiten können. Zusätzlich hatte ich entdeckt, dass es bei dieser Online-Version Figuren - unterschiedlichste Personen in unterschiedlichsten Haltungen - zur Verfügung stehen, die auf die Folien eingefügt werden können. Hier sollen kurz zwei der realisierten Anwendungen dieses Arrangements beschrieben werden.

Für den ersten kurzen Einstieg lasse ich das System zufällig Gruppen aus 2-3 Studierenden erstellen. Hier tauschen sie sich zunächst dazu aus, was sie schon zum Psychodrama wissen und was sie erwarten.

Als soziometrischen vertiefenden Einstieg werden erneut zufällig Kleingruppen gebildet, dieses Mal aus je 5-6 Studierenden. Jede Gruppe hat eine eigene OnlineFolie zur Verfügung. Auf dieser Folie ist eine Bühne angedeutet und es stehen „Figuren“ bereit. Hier kann jedeR eine als Alter Ego auswählen und diese auf der Bühne platzieren. Auf der Bühne stehen Kompetenzen auf virtuellen Moderationskarten geschrieben, die für SozialarbeiterInnen als relevant erachtet werden (AIEJI 2005). Nun sind die TeilnehmerInnen eingeladen, ihr Alter Ego auf die Kompetenz zu schieben, die eine besondere persönliche Stärke darstellt. Im Anschluss erfolgt ein Austausch über das Erleben während der Wahl sowie Gemeinsamkeiten und Unterschiede. Dies wird dann in der gesamten Gruppe wieder zusammengetragen.

Um eine Idee psychodramatischer Aktion zu vermitteln wird eine soziodramatische Ebene gewählt. Es soll pro Gruppe eine ,typische Problemlage“ als Szene auf der virtuellen Bühne dargestellt werden, denen SozialarbeiterInnen häufig begegnen. Zunächst richten die Studierenden die Bühne ein, wählen eine Figur als hypothetischE ProtagonistIn (z. B. das Kind, dass zwischen seinen Eltern nach der Scheidung steht) und erkunden dann über ,Doppeln“ das innere Erleben der prototypischen Charaktere und anderen Entitäten (z. B. ,das Jugendamt“). Ziel ist es, ein komplexes Bild der psychischen und sozialen Dynamiken zu erhalten.

\subsection{Momente und Atmosphäre}

Gleich zu Beginn kehrt eine Person spontan aus der digitalen Zweiergruppe in den Hauptraum zurück und benennt Gründe, warum es nicht möglich sei, mit der zufällig zugeteilten Person zu sprechen. Von der abgewählten Person erhalte ich gleichzeitig ein digitales Signal, dass um „Hilfe“ durch den Veranstalter - den Host - bittet. Ich teile beide zu anderen Zweiergruppen zu, die dadurch zu Dreiergruppen werden.

In den Kleingruppen entstehen interessante „typische Szenen“ aus dem Alltag der Sozialen Arbeit und viele Studierende melden zurück, dass eine vorsichtige Annäherung an die einzelnen Rollen gelungen sei und insbesondere Ambivalenzen an verschiedenen Stellen aufgespürt werden konnten. Einige Kameras bleiben 
ausgeschaltet und Personen stumm. Oft wird benannt, dass ein Respekt vor dem Psychodrama entstanden sei, wenn dies mit echten Menschen durchgeführt würde. Gleichzeitig wird die Sehnsucht benannt, genau das zu erleben. Eine Sehnsucht nach Begegnung im analogen Raum (s. unten).

$\mathrm{Ab}$ einem gewissen Zeitpunkt erscheint es mir plötzlich als seien nach und nach die Kameras ausgeschaltet worden, als seien Lichter ausgegangen. Ich schaue ins Dunkel. Ich habe private Nachrichten erhalten, dass manche sich unwohl fühlen in der Gruppenzusammensetzung und mich beschäftigt, wie dies in die gesamte Gruppe eingebracht werden könnte. Zum Abschluss sprechen die Studierenden an, dass es auch ein Gefühl von frei flottierender Bedrohlichkeit gibt. Für manche macht sich dies an einzelnen KommilitonInnen fest, für andere an einer nicht kontrollierbaren, potenziellen Veröffentlichung durch die Videokonferenztechnologie. Ein Misstrauen. Plötzlich ergreift mich auch dieses Misstrauen und ich spüre stark, dass ich die ganze Zeit meine Kamera angeschaltet gelassen habe. Für mich stellt sich die Herausforderung mit fehlender nonverbaler und zusätzlicher verbaler Kommunikation die Gruppe zu verstehen.

\section{3 Überlegungen im Rückblick}

Im Hinblick auf das einführend beschriebene Konzept der Affordanzen digitaler Medien möchte ich darüber nachdenken, wie sich eine verwendete Software auf die Ermöglichung und Verhinderung der Kontaktaufnahme im Seminarkontext ausgewirkt haben könnte. Dabei soll vor diesem Hintergrund exemplarisch am Beispiel von „Zoom“ (mit dessen Möglichkeiten, wie sie 2020 bestanden) darüber reflektiert werden, wie gruppenbezogene Entscheidungen durch mich als Dozent auch als eine „Verführung“ durch Affordanzen auf soziometrischer Ebene verstanden werden kann.

Die Software bietet einen virtuellen Hauptraum, mit Möglichkeiten zur Präsentation. Die anderen Personen können in verschiedenen Formaten ein- und ausgeblendet werden. Dazu kommt die Möglichkeit, die eigene Kamera und das eigene Mikrofon auszuschalten. Man sieht also z.T. nur die sprechende Person oder eine Auswahl von wenigen Video-,,Kacheln“. Diese Auswahl scheint durch einen Algorithmus bestimmt. Sie kann nicht direkt nachvollzogen oder beeinflusst werden. Anders als in einer Gruppe im realen Raum - im Stuhlkreis - weiß also keiner vom anderen verlässlich, ob und wenn ja, wohin er oder sie gerade schaut. Als Gruppenleitung kann ich mich nicht darauf beziehen. Stelle ich mir also vor, dass ich selbst die Kamera ausschalte, könnte es sein, dass jemand dennoch auf die mich repräsentierende, dunkle Kachel schaut. Selbst wenn ich mein Videobild mit der Gruppe teile, können einzelne mich in ihrer gewählten Ansicht u. U. gar nicht sehen etc. Gleiches gilt für den Ton. Findet hinter dem stumm geschalteten Mikrofon vielleicht gerade ein Familienstreit im „Homeoffice“ statt, pfeift der Wasserkessel oder geht die Klospülung? Alles ist denkbar. Ich frage mich, wie unterscheidet sich dies von Menschen in einer analogen (Psychodrama-)Gruppe, die innerlich abschweifen, häufig zur Wasserflasche greifen oder den Raum für einen Toilettenbesuch verlassen? Zunächst einmal hat niemand ,einen Platz“. Es ist nicht möglich zu entscheiden, neben wem das eigene Bild - selbst wenn es dunkel bleibt - erscheinen soll und neben 
welchem nicht. Von Anbeginn ist es soziometrisches Roulette und selbst wenn ich Kamera und Ton ausschalte, kann ich virtuell zwischen Personen eingereiht werden, die mir - real oder projektiv aufgeladen - nicht in meiner Nähe haben möchte. Zudem können Distanzen nicht analog reguliert werden; selbst ein Zurückrücken mit dem Stuhl ist nicht möglich.

Es gibt die technische Möglichkeit, spontan Kleingruppen unterschiedlicher Personenanzahl zu erstellen - zufällig durch die Software oder selbstgewählt manuell. Die Teilnehmenden werden auf einen Klick in die Kleingruppen verschoben und finden sich in einem eigenen virtuellen Raum mit den gleichen Darstellungsmöglichkeiten des „Hauptraums“ wieder. Sie können selbständig die Kleingruppe verlassen und in den Hauptraum zurückkehren. Sie können die DozentInnen aber auch um Hilfe bitten, d.h. einen Besuch im Raum der Kleingruppe anfordern. Die "Gastgeber“ des digitalen Raums können diese Kleingruppen dann besuchen und wieder verlassen und zwischendurch Textnachrichten in alle Kleingruppen gleichzeitig versenden. Auf diese kann nicht direkt geantwortet werden.

Die Möglichkeit, spontan Paare und kleine Gruppen zu bilden erschien mir von Anbeginn gut und damit verführerisch. Ein sicher auch vom Genuss der eigenen Macht als „Gastgeber“ beflügeltes Moment ist die Möglichkeit, ,endlich“ das ,,nervige" Diskutieren in Seminargruppen zu vermeiden, wer sich mit wem zusammentut. Gleichzeitig können wir durch den Kontrast mit der virtuellen Welt noch einmal besser verstehen, was spontane Paar- oder Gruppenbildung im Realen ausmacht. Es ist nicht Zufall, sondern Tele. Das sich Verwehren einer teilnehmenden Person mit einer bestimmten anderen in einer Zweiergruppe zu sprechen, sagt also nicht nur etwas über diese Person(en-Konstellation). Sie kann auch helfen zu verstehen, dass in dieser virtuellen Umgebung versucht werden kann, über große Strecken das Wirken von Teleprozessen auszuklammern. Und meine Überlegungen an dieser Stelle lassen mich erst im Nachhinein verstehen, wie hier die Verführung dieser digitalen Affordanzen mitgewirkt haben könnte: „In diesem Tool kann man zufällige Kleingruppen bilden, also bilde ich zufällig Kleingruppen, weil es eben geht“. An dieser Stelle scheinen zwei Aspekte von besonderer (praktischer) Bedeutung. Zum einen lässt sich der Verführung durch (Selbst-)Erfahrung mit dem jeweiligen digitalen Werkzeug und durch Reflexion eigener unbewusster Anteile der konkreten Verführbarkeit begegnen. D.h. wenn ich verstehen kann, warum ich eine bestimmte Funktion - in diesem Beispiel der Button zum Bilden zufälliger Kleingruppen immer wieder (auch unpassend) benutze, bin ich praktisch in der Lage diese Möglichkeit genauer auf den Lehr-Lernprozess abzustimmen. Dann nämlich - und dies ist der zweite Punkt - kann ich als gezielte intervenieren und Kleingruppen zufällig oder nach einem Kriterium bilden (lassen). In realen Seminargruppen beginne ich oft mit Kleingruppen aus SitznachbarInnen, der soziometrischen Annahme folgend, dass hier Tele bereits gewirkt hat. Später folgen zufällige Gruppen, um unerwarteten Austausch zu ermöglichen. Ähnlich konnte also ein solch gestufter Prozess, z. B. in Zoom nachempfunden werden.

Wenn wir uns nun fragen, was es für die Gesamtgruppe bedeutet, wenn in ,,machtvoller“ Weise die gastgebene Person - die oder der „Host“ - mit der Software soziometrische Entscheidung übernimmt. Es ist etwas Anderes als in einer Gruppe die Teilnehmenden zu bitten, sich spontan zusammenzutun für einen Austausch. 
Spontan ist unter der Annahme von Teleprozessen niemals zufällig! Wenn also bei spontaner Wahl wir davon ausgehen können, das wir uns in Richtung der forschenden Haltung soziometrischer Tests bewegen, die uns etwas über die Tiefenstruktur der Gruppe erkennen lassen (vgl. Stadler 2013), so können wir annehmen, dass die digital-intransparente Zuordnung ein bestimmtes Soziogramm nahelegt. Es entstehen Teilgruppen, die alle sternförmig mit dem „Host“ verbunden sind, die Verbindung aber eigentlich vermittelt durch das digitale Medium gehalten wird. Alle beteiligten richten also einen inneren fragenden Blick auf die Software, die auf unheimliche Weise und allmächtig zu handeln scheint, bzw. zu befähigen zu beschränken.

Psychoanalytisch gedacht, könnte man die These aufstellen, dass sowohl die Gruppe(nmitglieder), als auch Host in eine regressive Tendenz gezogen werden. Psychodramatisch gedacht, ergeben sich unvereinbare Rollenerwartungen: Während der Austausch in der Kleingruppe, die „ohne Zutun“ des Hosts gebildet wurde, die Autonomie der Studierenden stärken soll, führt gleichzeitig die Art und Weise der zufälligen Gruppenbildung zu einem Dilemma. Die Studierenden finden sich durch ein allmächtig erscheinendes Handeln des Host in dieser Konstellation wieder. Versteht man das Setting so, liegt es für Gruppenmitglieder nah, bei Verunsicherung sich desselben Weges rückwärtig zu bedienen, also individuelle Nachrichten an den Host zu senden, was durch Funktionen, diesen zur Hilfe zu rufen. Dies wird auf der Ebene der Affordanzen durch entsprechende Möglichkeiten der Software gleichzeitig befördert. Darüber hinaus fehlen Möglichkeiten die Wahlen der Gruppen in den Pausen oder Wortfetzen aus den Seitengesprächen zu hören und einzubeziehen. Das digitale „Tuscheln“, das in Privatnachrichten zwischen den Teilnehmenden phantasiert werden kann, ist per digitalem Medium stumm und unsichtbar.

\section{Kann es Begegnung im virtuellen Raum geben? Soll es das?}

In diesem Beitrag wird die Verwendung digitaler Medien in Lehr-Lernkontexten beschrieben und vor dem Hintergrund psychodramatischer Konzepte reflektiert. Dabei wurde der Prämisse gefolgt, dass digitalen Medien Affordanzen zu eigen sind, die spezifische Prozesse des Denkens, Lernens oder der Interaktion begünstigen und andere verhindern. Am Beispiel eines Seminars via Videokonferenz wurde skizziert, dass wir davon ausgehen können, das dies auch auf der soziometrischen Ebene zu bedenken ist. Bis hierher wurde somit ein Fokus auf mögliche spezifische Einflüsse gelegt. Ich möchte mich daher noch kurz einem weiteren Aspekt zuwenden, nämlich der Frage, wie Lehr-Lern-Kontexte ganz grundlegend psychodramatisch konzeptualisiert werden können und wie die digitale Kommunikation im Home-Schooling und Home-Office letztlich zu einem Paradoxon von Nähe und Distanz führt. Hier scheint es wichtig noch einmal den prinzipiellen Charakter des Konzepts der Affordanzen zu betonen. Gerade in den letzen Monaten haben die konkreten digitalen Werkzeuge viele Überarbeitungen erfahren. Das Zoom von heute ist schon nicht mehr das des Fallbeispiels in diesem Artikel. Es wäre also im Detail unzureichend bis inkorrekt zu sagen, das Seminar in diesem Beispiel wäre mit ,Zoom“ durchgeführt worden. Die Reflexion der aktuell vorhanden Affordanzen stellt das Bemühen dar, den Bezug zwischen einzelnen Funktionen und dem Aufforderungscharakter für ,den Men- 
schen“, „die Gruppe“ etc. konzeptuell zu fassen. Dieser Artikel stellt also explizit keine Handlungsanweisung konkret für Zoom dar!

Moreno beschreibt Begegnung als „Zusammensein, Zusammentreffen, Berührung zweier Körper, Sehen und Beobachten, Berühren, Einfühlen, Teilen und Lieben, Verständigung miteinander, intuitives Erkennen durch Schweigen oder Bewegung, Sprache oder Gesten, Kuß oder Umarmung, Einswerden - una cum uno.“ (1997 [1959], S. 54). Andere Kontaktformen nennt er „Konserven“ (ebd.). Gleichzeitig beschreibt er den Kontakt, der auf sozialen Rollen beruht, wie der zwischen TherpeutIn und KlientIn oder LehrerIn und SchülerIn als ,begrenzten Kontakt“ (ebd.). Welche Rolle spielt also Begegnung in begrenzten Kontakten? Ich verstehe Moreno hier so, dass das Zusammentreffen in konservierten (komplementären) Rollen das soziale Miteinander dahingehend strukturiert, als dass Verhaltensmuster zu einem gewissen Grad erwartbar, vorhersagbar und planbar werden. Das sich dies auf die darum herum entstehenden organisationalen Strukturen fortschreiben lässt, soll hier nur erwähnt werden. Was also im Unterricht, in einer Vorlesung, in einem Seminar geschieht, kreist um geteilte Vorstellungen und Rollenerwartungen. Bezieht man die bisherigen Überlegungen zu digitalen Medien mit ein, so können wir davon ausgehen, dass die Affordanzen digitaler Medien - bzw. die individuellen Vorstellungen davon (mentale Modelle) diese Konserven beeinflussen. Einerseits kann darin eine Erwärmung entdeckt werden, so dass sich Spontaneitätslagen ergeben, um die Rolle der Lehrenden und der Lernenden und den Kontakt zwischen ihnen kreativ neu zu gestalten z.B. von der Meister-Schüler hin zur Entdecker-Lerncoach-Konserve. Andererseits sind Prozesse zu bemerken, die Entstehung von Spontaneität und Kreativität vermeiden. Das Konservative bietet angesichts der emergenten und flexiblen Möglichkeiten der digitalen Technologie Sicherheit: Wenn ich schon nicht weiß, wozu mich dieses digitale Werkzeug alles „,verleiten“ könnte, dann konserviere ich möglichst den bekannten begrenzten Kontakt und vermeide Begegnung.

In diesem Zusammenhang sind auch die Ausführungen Morenos im Anschluss an die oben zitierten interessant. Er bezieht sich auf die Anfänge der Gruppenpsychotherapie und beschreibt, dass hier zu Beginn „Familien-Gruppen-Psychotherapie in situ“ im Heim der Familien stand. Somit hätten diese ersten Therapien in ,wirklichen Lebenslagen" (ebd., Hervorhebungen im Original) stattgefunden. Mir scheint als würde Moreno darum ringen, wie man sich (als TherapeutIn) an die zuvor idealisiert dargestellte Begegnung auch in begrenzten Konserven-Kontakten annähern kann. Lässt man einmal die Frage beiseite, ob in Szenarien digitalen Lehrens und Lernens immer Begegnung ein Ziel sein sollte, so springt doch in dem hier verhandelten Aspekt der Digitalisierung ein Paradoxon ins Auge. Kehren wir zu der Videokonferenz als Rahmen zurück. Hier findet der begrenzte Kontakt virtuell statt und viele der von Moreno beschriebenen Aspekte der körperlichen Wahrnehmung fallen fort; nicht einmal ein Schweigen ist immer ein Schweigen. Wenn Teilnehmende bei ausgeschaltetem Mikrofon zu jemand anderem sprechen. Auch das Hinoder Fortschauen ist dauerhaft ambivalent, z. B. wenn Personen mit ausgeschalteter Kamera weiter beobachten aber anderen den Blick auf sich selbst verwehren. Das eigentlich Paradoxe aber ergibt sich, wenn man darüber nachdenkt, dass nun viele der Beteiligten zuhause, in ihrer wirklichen Lebenslage an der Gruppe teilnehmen. Von dieser Warte aus betrachtet wird der begrenzte Kontakt aufgebrochen und es 
gibt eine andere Verführung, und zwar die hin zur Begegnung in situ des eigenen Heims. Nun kann aber gerade große Verführung besonderen Widerstand erzeugen. Dies stellt aus meiner Sicht eine grundlegende Forschungsfrage, nämlich welche Dynamiken herrschen, wenn ich gleichzeitig allein zuhause und virtuell in einer Gruppe bin. Was trägt zur Erwärmung bei, was bewirkt eher eine Verfestigung von Konserven? Braucht es mehr oder andere Erwärmung? Braucht es hier vielleicht auch einen Cool-Down, also einen Übergang in die Realität des eigenen Zimmers zurück? Konzeptuell stellt sich die Frage, wo hier Surplus-Reality entsteht - im realen Zimmer, in dem ich virtuell in eine Gruppe begeben kann oder eher im virtuellen Raum, wenn dort nach und nach etwas Gemeinsames entsteht oder beides eine virtuelle Surplus Realität, die in der realen geschachtelt entsteht. Hierin verbirgt sich möglicherweise eine weitere Perspektive, die beim Home-Schooling oder Home-Office, wo ich nicht vornehmlich in privaten Rollen handele, an Bedeutung zunimmt. Hier gilt es also sich mit Fragen der Nähe-Distanz-Regulation zu beschäftigen, welche andere Fragen sind als jene, die sich im begrenzten Kontakt in einem analogen Setting stellen.

\section{Fazit}

Ausgehend von einem kurzen Einblick in aktuelle Konzepte der pädagogisch-psychologischen Forschung zu Fragen des Unterrichtens wurden Ansätze beschrieben, die Rolle digitaler Medien in diesem Kontext zu verstehen. Mit einem besonderen Fokus auf das flexible und emergente der Eigenschaften aktueller digitaler Medien wurde das Konzept des Aufforderungscharakters - der Affordanzen - dieser Medien eingeführt und betont, dass dieser auch in Abhängigkeit der korrespondierenden mentalen Modelle der NutzerInnen variiert. Daraus resultieren spezielle Anforderungen für Lehrende, was an einem Beispiel aus einem als Videokonferenz Online durchgeführten Seminars zur Einführung in das Psychodrama illustriert wurde. Das Konzept der Affordanzen wurde auf die soziometrische Ebene übertragen und es konnten verschiedene Verführungen rekonstruiert werden, das digitale Werkzeug als intransparenten und damit machtvollen Akteur im Soziogramm zu verstehen. Dabei spielen verschiedene Bemühungen der Beteiligten eine Rolle, die Wahlen auf Basis von Teleprozessen (wieder) herzustellen. Abschließend lässt sich also als These formulieren, dass wir Affordanzen digitaler Medien nicht nur auf einer kognitiven und sozialen, handlungsnahen Ebene im Sinne fachlichen Lernens verstehen können, sondern auch ihren Einfluss auf Begrenzung und Ermöglichung soziometrischer Wahlen und letztlich von Begegnung betrachten sollten.

Danksagung gilt den Studierenden für Ihre Offenheit sich auf ein Online-Kennenlernen des Psychodramas einzulassen.

Funding Open Access funding enabled and organized by Projekt DEAL.

Open Access Dieser Artikel wird unter der Creative Commons Namensnennung 4.0 International Lizenz veröffentlicht, welche die Nutzung, Vervielfältigung, Bearbeitung, Verbreitung und Wiedergabe in jeglichem Medium und Format erlaubt, sofern Sie den/die ursprünglichen Autor(en) und die Quelle ord- 
nungsgemäß nennen, einen Link zur Creative Commons Lizenz beifügen und angeben, ob Änderungen vorgenommen wurden.

Die in diesem Artikel enthaltenen Bilder und sonstiges Drittmaterial unterliegen ebenfalls der genannten Creative Commons Lizenz, sofern sich aus der Abbildungslegende nichts anderes ergibt. Sofern das betreffende Material nicht unter der genannten Creative Commons Lizenz steht und die betreffende Handlung nicht nach gesetzlichen Vorschriften erlaubt ist, ist für die oben aufgeführten Weiterverwendungen des Materials die Einwilligung des jeweiligen Rechteinhabers einzuholen.

Weitere Details zur Lizenz entnehmen Sie bitte der Lizenzinformation auf http://creativecommons.org/ licenses/by/4.0/deed.de.

\section{Literatur}

AIEJI (2005). Die professionellen Kompetenzen von Sozialpädagogen/innen/innen. Ein konzeptioneller Rahmen. International Association of Social Educators, AIEJI. http://www.vegjd.de/downloads/ AIEJI\%20conceptual\%20framework_DE.pdf. Zugegriffen: 20.05.2021.

Angeli, C., \& Valanides, N. (2018). Knowledge base for information and communication technology in education. In J. Voogt, G. Knezek, R. Christensen \& K.-W. Lai (Hrsg.), Second handbook of information technology in primary and secondary education (S. 397-413). Berlin Heidelberg: Springer. https://doi.org/10.1007/978-3-319-71054-9_26.

Baumert, J., \& Kunter, M. (2013). The COACTIV model of teachers' professional competence. In M. Kunter, J. Baumert, W. Blum, U. Klusmann, S. Krauss \& M. Neubrand (Hrsg.), Cognitive activation in the mathematics classroom and professional competence of teachers (S. 25-48). New York: Springer. https://doi.org/10.1007/978-1-4614-5149-5_2.

Blömeke, S. (2000). Medienpädagogische Kompetenz. Theoretische und empirische Fundierung eines zentralen Elements der Lehrerausbildung. : KoPäd.

Chi, M.T.H. (2000). Self-explaining expository texts: the dual processes of generating inferences and repairing mental models. In Advances in instructional psychology (S. 161-238). : Lawrence Erlbaum.

Clark, R.E. (1983). Reconsidering research on learning from media. Review of Educational Research, 53, 445-459.

Gershoni, J. (2016). Highlighting sociometry in the psychodramatic process. Zeitschrift für Psychodrama und Soziometrie, 15(2), 353-357. https://doi.org/10.1007/s11620-016-0351-z.

Gibson, J. J. (1982). Wahrnehmung und Umwelt. München u.a.: Urban \& Schwarzenberg.

Helmke, A. (2009). Unterrichtsqualität und Lehrerprofessionalität. Diagnose, Evaluation und Verbesserung des Unterrichts (2. Aufl.). : Kallmeyersche Verlagsbuchhandlung.

Johnson-Laird, P.N. (1983). Mental models: towards a cognitive science of language, inference and consciousness. Cambridge: Univerisity Press.

Klieme, E., \& Rakoczy, K. (2008). Empirische Unterrichtsforschung und Fachdidaktik. Outcome-orientierte Messung und Prozessqualität des Unterrichts. Bd. 19.

Kozma, R. (1994). A reply: media and method. Educational Technology Research and Development, 42, $7-19$.

Krauskopf, K. (2019). Die professionelle Begegnung - ein Workshop zur professionsorientierten biographischen Reflexion für Lehramtsstudierende auf der Grundlade psychodramatischer Rollentheorie. In U. Graf \& T. Iwers (Hrsg.), Beziehungen bilden. Wertschätzende Interaktionsgestaltung in pädagogischen Handlungsfeldern (S. 57-66). Bad Heilbrunn: Julius Klinkhardt.

Krauskopf, K., \& Teke, G. (2018). Begegnung mit dem vermeintlich Sonderbaren. Zeitschrift für Psychodrama und Soziometrie, 17(2), 261-271. https://doi.org/10.1007/s11620-018-0445-x.

Krauskopf, K., Zahn, C., \& Hesse, F.W. (2012). Leveraging the affordances of Youtube: the role of pedagogical knowledge and mental models of technology functions for lesson planning with technology. Computers \& Education, 58(4), 1194-1206. https://doi.org/10.1016/j.compedu.2011.12.010.

Krauskopf, K., Zahn, C., \& Hesse, F.W. (2015). Cognitive processes underlying TPCK: mental models, cognitive transformation, and meta-conceptual awareness. In C. Angeli \& N. Valanides (Hrsg.), Technological pedagogical content knowledge (S. 41-61). New York: Springer. https://doi.org/10.1007/ 978-1-4899-8080-9_3.

Krauskopf, K., Zahn, C., \& Hesse, F.W. (2020). Conceptualizing (pre-service) teachers' professional knowledge for complex domains. The case of technology and heterogeneity. In T. Lehmann (Hrsg.), 
International perspectives on knowledge integration: theory, research, and good practice in pre-service teacher and higher education (S. 31-58). Brill: Sense Publishers.

Kulik, J. (2003). Effects of using instructional technology in elementary and secondary schools: What controlled evaluation studies say. Arlington: SRI International. www.sri.com/policy/csted/reports/ sandt/it/Kulik_ITinK-12_Main_Report.pdf

Mishra, P., \& Koehler, M. J. (2006). Technological pedagogical content knowledge: a new framework for teacher knowledge. Teachers College Record, 108(6), 1017-1054.

Moreno, J.L. (1961). The role concept, a bridge between psychiatry and sociology. American Journal of Psychiatry, 118(6), 518-523. https://doi.org/10.1176/ajp.118.6.518.

Moreno, J. L. (1997). Gruppenpsychotherapie und Psychodrama (5. Aufl.). Stuttgart: Thieme.

Stadler, C. (2013). Was ist Soziometrie? In C. Stadler (Hrsg.), Soziometrie. Wiesbaden: Springer VS. https://doi.org/10.1007/978-3-531-18981-9_2.

Suthers, D. (2006). Technology affordances for intersubjective meaning making: A research agenda for CSCL. International Journal of Computer-Supported Collaborative Learning, 1, (3), 315-337

Valanides, N. (2018). Technological tools: from technical affordances to educational affordances. Problems of Education in the 21st Century, 76(2), 116.

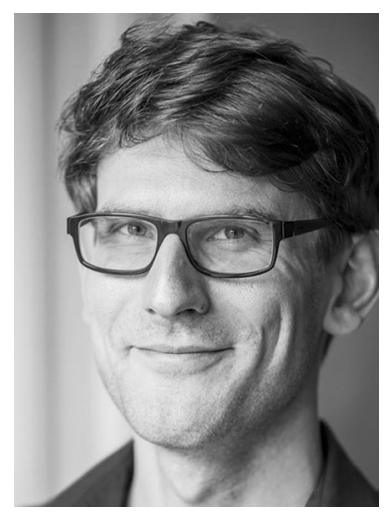

Karsten Krauskopf 1979, Dr. rer. nat., Dipl.-Psych., B.A., Psychodrama-Therapeut (DFP, FEPTO) ist Professor für Psychologie in der Sozialen Arbeit an der FH Potsdam, selbständig als Prozessbegleiter und Supervisor tätig sowie in Weiterbildung zum Psychoanalytiker und analytischen Kinder- und Jugendlichen-Psychotherapeuten. 